\title{
The DMC Contribution in the Human Absorption in an Indoor Environment
}

\author{
Aliou Bamba*, Wout Joseph, Günter Vermeeren, Emmeric Tanghe and Luc Martens Ghent \\ University / IBBT - Department of Information Technology \\ Gaston Crommenlaan 8 box 201, B-9050 Ghent-Belgium \\ Fax: +3293314 899, Tel: +3293314908 \\ *Email: aliou.bamba@intec.ugent.be
}

\begin{abstract}
Room electromagnetics theory is applied to determine the influence of the diffuse multipath components (DMC) in the human specific absorption rate at 2.8 GHz. The whole-body absorption is determined for an exposure to a Line Of Sight (LOS) component plus the DMC.

Both experimental and numerical results show that the contribution of the DMC in the whole-body absorption can not be neglected. The whole-body specific absorption rate (SAR) may be due up to $90 \%$ to the DMC in an indoor environment.
\end{abstract}

\section{INTRODUCTION}

The International Commission on Non-Ionizing Radiation Protection (ICNIRP) [1] defines the Specific energy Absorption Rate (SAR) as a basic restriction for human exposure in the radio frequency (RF) band. Various works based on numerical computations such as the Finite-Difference Time-Domain (FDTD) method have assessed the SAR in phantoms [2]-[4], but the whole-body SAR has been calculated in these papers for a set of plane waves exposure.

The presence of the DMC is reported recently in [5], and it has gained interest in the wireless channels parameters estimators community. The characteristics of DMC in an indoor environment are investigated in [6], where it turns out that the contribution of the DMC to the total power may range from $10 \%$ to $95 \%$. Hence, the DMC power may contribute to the absorption in human in a realistic environment. This matter is not yet adressed in the literature. The DMC contribution in the absorption is determined experimentally and atferwards numerically using Finite-Difference TimeDomain (FDTD) simulation. The outline of this abstract is as follows: In Section 2, we present the materials and the methods. Section 3 is devoted to the results from measurements and numerical computation. Conclusion is drawn in Section 4.

\section{Methods}

A virtual Multiple Input Multiple Output (MIMO) channel sounder is used to probe the channel. For the present work, the Tx location is fixed and the $\mathrm{Rx}$ is positioned at $100(10 \times 10)$ different locations forming a virtual uniform rectangular array. Both antennas are polarized vertically and positioned at a height of about $1.80 \mathrm{~m}$ above the ground. Five cylindrical phantoms in Polyvinyl chloride (PVC) (height $=150 \mathrm{~cm}$, diameter $=24 \mathrm{~cm}$ ) are used to assess the absorption cross section of one phantom (ACS). Phantoms were filled with water with following properties: $\sigma=1.58 \Omega^{-1} / \mathrm{m}$ (conductivity), $\epsilon_{r}=77.32$ (relative permittivity) and $\rho=1000 \mathrm{~kg} / \mathrm{m}^{3}$ (mass density). The measurements are performed in an office room of about $360 \mathrm{~m}^{3}$.

Experimentally, the $\mathrm{SAR}_{w b}^{\text {meas }}$ of the phantom is determined from its absorption cross section (ACS in $\mathrm{m}^{2}$ ) and the incident power densities, i.e., line of sight (LOS) and DMC (in $\mathrm{W} / \mathrm{m}^{2}$ ). Room electromagnetics theory is applied in [7] to assess the ACS of humans. The same method is used here for the ACS assessment of the phantom. Since the LOS component amplitude is measured, its power density is easily determined [8], [9] and the DMC power density is investigated in [10]. As already stated, the contribution of the DMC power density in the total power may vary drastically. Fig. 1 shows the relation between the LOS and DMC power densities in our investigated indoor environment [9]. The $\mathrm{SAR}_{w b}$ is calculated for 3 scenarios:

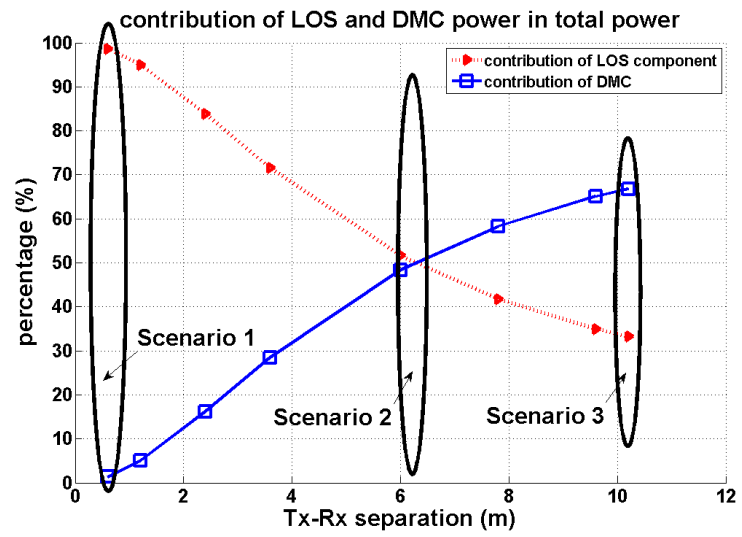

Fig. 1. Contribution of coherent and DMC in the total power density int he investigated room 
1) the phantom is in the vicinity of the transmitter, i.e., where the LOS component is dominating

2) the phantom is located at the reverberation distance (where the LOS and the DMC power densities are the same, about $6 \mathrm{~m}$ in Fig. 1)

3) the phantom is far away from the transmitter, i.e., where the LOS component can be neglected compared to the DMC component

The experimentally measured whole-body SAR is expressed as follows:

$$
\begin{aligned}
S A R_{w b}^{\text {meas }} & =S A R_{w b}^{s p e c}+S A R_{w b}^{d i f} \\
& =\frac{A C S}{m}\left(I_{d i f}+\frac{1}{\pi} \sum_{k=1}^{N_{s c}} I_{s c}^{k}\right)
\end{aligned}
$$

where $S A R_{w b}^{s p e c}, S A R_{w b}^{d i f}, I_{d i f f}, I_{s c}^{k}, N_{s c}$ represent the absorption rate due to the specular components, the absorption rate due to the DMC, the total power density of the DMC, the power density of the $k^{t h}$ specular path and the total number of specular paths, respectively. Note that for this work, only the first specular component (LOS) is taken into account $\left(\mathrm{N}_{s c}=1\right)$.

For the numerical calculations, the FDTD method (SEMCAD-X [11]) has been used to compute the averaged $\mathrm{SAR}_{w b}^{f d t d}$ absorbed by the phantom. The same dielectric properties are used during the simulations.

The DMC are modeled with plane waves in SEMCAD$\mathrm{X}$ as follows: an exponential decay, a white azimuthal spectrum, uniformely distributed delays in time domain, an elevation in the horizontal plane $\left(\theta=90^{\circ}\right)$. The power densites (in $\mathrm{mW} / \mathrm{m}^{2}$ ) used are summarized in Table. I.

\begin{tabular}{|c|l|l|l|}
\hline Phantom-Tx & $\begin{array}{l}\text { LOS } \\
\text { power }\end{array}$ & $\begin{array}{l}\text { DMC } \\
\text { power }\end{array}$ & $\begin{array}{l}\text { Total } \\
\text { power }\end{array}$ \\
\hline $0.60 \mathrm{~m}$ (scenario 1) & 694.40 & 2.90 & 697.30 \\
\hline $6 \mathrm{~m}$ (scenario 2) & 2.20 & 2.1 & 4.30 \\
\hline $10.20 \mathrm{~m}$ (scenario 3) & 0.40 & 1.60 & 2 \\
\hline
\end{tabular}

TABLE I

POWER DENSITIES ACCORDING TO THE SCENARIOS

\section{RESULTS}

Table II shows the experimentally measured $\mathrm{SAR}_{w b}$ values and the percentage of the DMC contribution. It can be seen that in the vicinity of the transmitter (scenario 1) the highest power density leads to the highest absorption rate. As the phantom is moved away from the transmitter, the $\mathrm{SAR}_{w b}^{\text {meas }}$ decreases drastically. Even tough the absorption decreases with increasing separation from the transmitter, the contribution of the DMC becomes more significant, varying from $1.30 \%$ (scenario 1) up to $92 \%$ (scenario 3 ). For the numerical simulation the DMC is modeled with $0,4,8,16,32,64$ and 72 plane waves to show

\begin{tabular}{|l|l|l|l|}
\cline { 2 - 4 } \multicolumn{1}{c|}{} & scenario 1 & scenario 2 & scenario 3 \\
\hline SAR $_{w b}^{\text {meas }}$ & $\begin{array}{l}1284.30 \\
(\mu \mathrm{W} / \mathrm{kg})\end{array}$ & $\begin{array}{l}16.10 \\
(\mu \mathrm{W} / \mathrm{kg})\end{array}$ & $\begin{array}{l}9.90 \\
(\mu \mathrm{W} / \mathrm{kg})\end{array}$ \\
\hline $\begin{array}{l}\text { Part of DMC } \\
\text { in SAR }\end{array}$ & $1.30 \%$ & $75 \%$ & $92.60 \%$ \\
\hline
\end{tabular}

TABLE II

$\mathrm{SAR}_{w b}^{\text {meas }}$ FOR DIFFERENT SCENARIOS

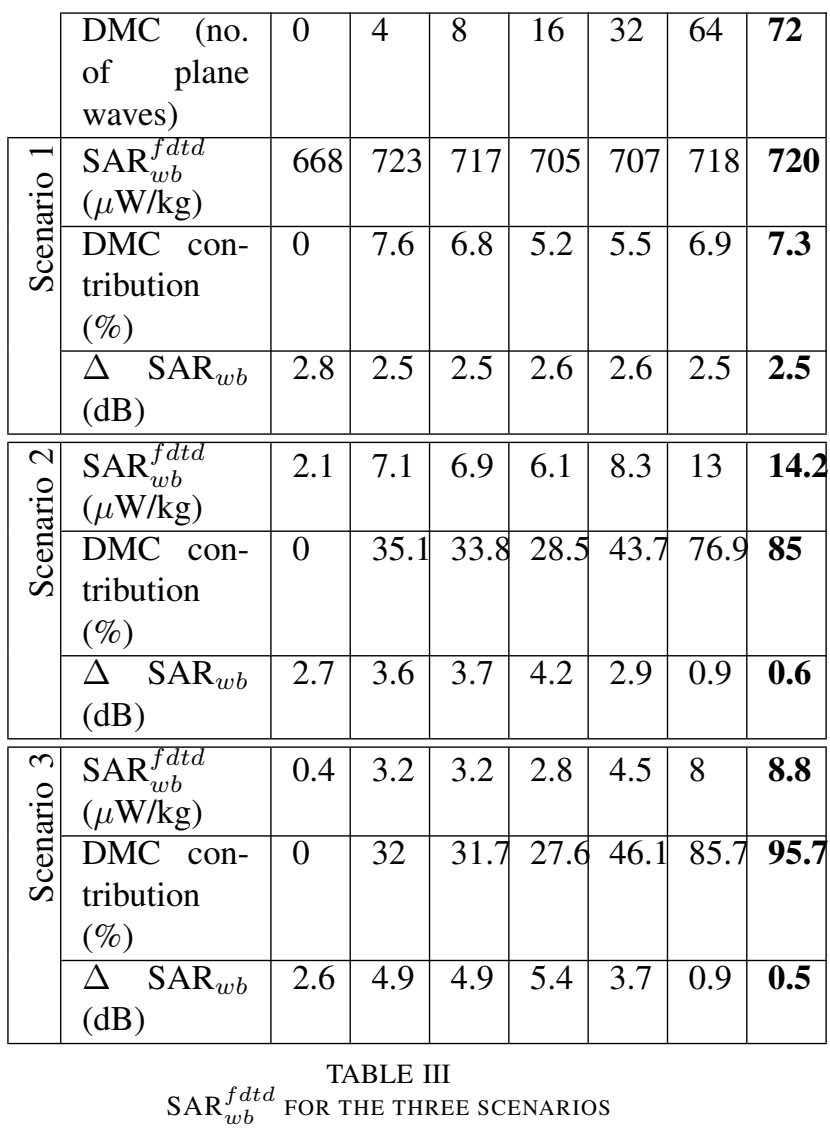

convergence and accuracy of the modeling. Table III lists the numerical results where the values in bold are the most accurate. Unfortunately, simulating with more plane waves was not possible, because of software and/or hardware limitations. Moreover Table III shows that from 64 planes waves on the results do not vary much and 72 planes waves satisfy.

$\Delta \mathrm{SAR}_{w b}$ is the relative error (in $\mathrm{dB}$ ) between the experimentally measured $\mathrm{SAR}_{w b}$ and the simulated $\mathrm{SAR}_{w b}$. The DMC contribution varies from $1.3 \%$ to $92 \%$ in the experimental SAR whereas it varies from $7.3 \%$ up to $95.7 \%$ for the numerical calculation, indicating the importance of the DMC in the total absorption. In the full paper, the methods and the results will be discussed in details. 


\section{CONCLUSION}

The DMC contribution in the whole-body specific absorption rate is studied in this paper. Even though the whole-body $\mathrm{SAR}_{w b}$ decreases with increasing separation from the transmitter, the contribution of the DMC in the absorption may reach up to $90 \%$. Experimental and numerical results agree very well as long as the plane wave assumption is valid (scenario 2 and 3 ). Checking compliance with the ICNIRP guidelines is not the purpose of the present paper, but the presented method can be used for this. We present a method to determine experimentally the whole-body SAR in real environments plus its validation via numerical simulations. In addition, the DMC contribution is highlighted. Comparison with the ICNIRP restrictions values requires also the knownledge of the duty cycle of the considering system.

\section{ACKNOWLEDGMENT}

The research leading to these results has received funding from the European Union's Seventh Framework Programme (FP7/2007-2013) under grant agreement no 244149. The research is also partly funded by the Fund for Scientific Research - Flanders (FWO-V, Belgium) project G.0325.11N.

Wout Joseph is a Post-Doctoral Fellow of the FWO-V (Research Foundation - Flanders).

\section{REFERENCES}

[1] Guidelines for limiting exposure to time-varying electric, magnetic, and electromagnetic fields (up to $300 \mathrm{GHz}$ ). Technica report, International Commission on Non-Ionizing Radiation Protection.

[2] P. J. Dimbylow. Fine resolution calculations of SAR in the human body for frequencies up to $3 \mathrm{GHz}$. Physics In Medicine And Biology, 47: pages 2835-2846, 2002.

[3] Günter Vermeeren, Wout Joseph and Luc Martens. Wholebody SAR in spheroidal adult and child phantoms in a realistic exposure environment. IEE Electronics Letters (44)13:, (44) 13: pages 790-791, 2008.

[4] Jianqing Wang, Osamu Fujiwara, Sachiko Kodera and Soichi Watanabe. FDTD calculation of whole-body average SAR in adult and child models for frequencies from $30 \mathrm{MHz}$ to $3 \mathrm{GHz}$. Physics In Medicine And Biology, 51: pages 4119-4127, 2006.

[5] Andreas Richter. Estimation of Radio Channel Parameters: Models and Algorithms. PhD thesis, Fakultt fr Elektrotechnik und Informationstechnik der Technischen Universitt Ilmenau, 2005.

[6] Juho Poutanen, Jussi Salmi, Katsuyuki Haneda, Veli-Matti Kolmonen, and Pertti Vainikainen. Angular and Shadowing Characteristics of Dense Multipath Components in Indoor Radio Channels. IEEE Transactions on Antennas and Propagation, 59: pages 245-253, 2011.

[7] A. Bamba, J. B. Andersen, W. Joseph, D. Plets, E. Tanghe, G. Vermeeren, J. O. Nielsen and L. Martens. Experimenta Specific Energy Absorption Rate Assessment from Absorption Cross Section Measurement for Far-field Exposure at 2-3 GHz. In in 33rd Annual Meeting of the Bioelectromagnetics Society, Halifax, CA, June 2011. (Accepted), 2011.

[8] Harald T. Friis. A note on a Simple Transmission Formula. Procedings of the I.R.E and Waves and Electrons, pages pages 254-256, May, 1946.
[9] A. Bamba, W. Joseph, J. B. Andersen, E. Tanghe, G. Vermeeren, D. Plets, J. O. Nielsen, and L. Martens. Experimental Assessment of Specific Absorption Rate Using Room Electromagnetics. IEEE Transactions on Electromagnetic Compatibility (accepted with minor revisions), 2011.

[10] Jørgen Bach Andersen, Kin Lien Chee, Martin Jacob, Gert Frølund Pedersen, Thomas Kürner. Reverberation and Absorption in an Aircraft Cabin with the Impact of Passengers. accepted for publication at Antennas and Propagation Transactions, 2011.

[11] Schmid \& Partner Engineering AG, SEMCAD-X. www.semcad.com. 